\title{
Gravitational Coupling and the Cosmological Constant
}

\author{
Yousef Bisabr* \\ Department of Physics, Shahid Rajaee Teacher Training University, Lavizan, Tehran 16788, Iran
}

\begin{abstract}
We deal with a dynamical mechanism in which a large cosmological constant, as suggested by inflationary scenarios, decays due to expansion of the universe. This mechanism has its origin in the gravitational coupling of the vacuum density. We assume that the vacuum couples anomalously to gravity that is the metric tensor that appears the gravitational part is not the same as that appears the matter part as suggested by weak equivalence principle. Instead, the two metric tensors are taken to be conformally related. We show that this provides a dynamical mechanism which works during expansion of the universe. We also consider some observational consequences of such a gravitational model.
\end{abstract}

Keywords: Gravity, Modified Gravity, The Cosmological Constant.

\section{Introduction}

There is a large discrepancy between observations and theoretical estimates on the vacuum energy density. This problem is known as the first cosmological constant problem (or the fine tuning problem) [1] [2]. The second one known as the coincidence problem [3] deals with the question that why the vacuum energy is the same order of magnitude of the matter density. There have been many attempts trying to resolve the fine tuning problem [2]. Most of them are based on the belief that the cosmological constant may not have such an extremely small value at all times. In fact, it has a large value at early times as suggested by inflationary models and there should exist a dynamical mechanism working during evolution of the universe which provides a cancelation of the vacuum energy density at late times [4].

In this work, we will consider the possibility that this cancelation mechanism is related to gravitational coupling of matter in a gravitational system. The matter part of a gravitational system is usually taken to be coupled with the metric which describes the gravitational part. This is a normal coupling which has its origin in the weak equivalence principle. Firstly, it should be pointed out that even though, observational evidences are fully consistent with

\footnotetext{
*e-mail: y-bisabr@srttu.edu.
} 
weak equivalence principle, the experiments that support the principle are recent and it is quite possible that the principle is violated during evolution of the universe. Secondly, the weak equivalence principle is a statement about the gravitational coupling of normal matter and there is no experimental constrains on gravitational coupling of the other forms of energy densities such as vacuum.

We will assume that the vacuum energy density does not couple normally to gravity but rather they couple abnormally or anomalously, that is the metrics that appear in the matter and the gravitational parts are different but conformally related [4]. As illustrations, we will consider such an anomalous gravitational coupling in $f(R)$ gravity [8] [9] and Brans-Dicke (BD) theory [10] and discuss the conditions that lead to alleviation of the cosmological constant problem.

\section{$2 \quad$ Field Equations}

We consider the action functional

$$
S=\int d^{4} x \sqrt{-g}\left\{\frac{M_{p}^{2}}{2} R-\frac{1}{2} g^{\mu \nu} \nabla_{\mu} \varphi \nabla_{\nu} \varphi-V(\varphi)\right\}+S_{m}\left(A^{2}(\varphi) g_{\mu \nu}, \psi\right)
$$

where $M_{p}^{-2} \equiv 8 \pi G, G$ is the gravitational constant and $S_{m}$ is the action of some matter field $\psi$. The function $A(\varphi)$ is a coupling function that characterizes coupling of the scalar field $\varphi$ with the matter sector. It defines anomalous gravitational coupling of matter characterized by the field $\psi$. In general, such a matter system does not respect the weak equivalence principle. However, the scalar field $\varphi$ can hide the anomalous coupling via chameleon mechanism [5] and pass local gravity experiments [6] [7].

Variation of this action with respect to the metric tensor $g^{\mu \nu}$, gives

$$
G_{\mu \nu}=M_{p}^{-2}\left(T_{\mu \nu}^{\varphi}+T_{\mu \nu}^{m}\right)
$$

where

$$
\begin{gathered}
T_{\mu \nu}^{\varphi}=\nabla_{\mu} \varphi \nabla_{\nu} \varphi-\frac{1}{2} g_{\mu \nu} \nabla^{\gamma} \varphi \nabla_{\gamma} \varphi-V(\varphi) g_{\mu \nu} \\
T_{\mu \nu}^{m}=\frac{-2}{\sqrt{-g}} \frac{\delta S_{m}}{\delta g^{\mu \nu}}
\end{gathered}
$$

are stress-tensors of the scalar field and the matter system. If we vary the action with respect to the scalar field $\varphi$, we obtain

$$
\square \varphi-\frac{d V(\varphi)}{d \varphi}=-\frac{\beta(\varphi)}{M_{p}} T^{m}
$$

or, equivalently,

$$
\nabla^{\mu} T_{\mu \nu}^{\varphi}=-\frac{\beta(\varphi)}{M_{p}} \nabla_{\nu} \varphi T^{m}
$$

where

$$
\beta(\varphi)=M_{p} \frac{d \ln A(\varphi)}{d \varphi}
$$


and $T^{m} \equiv g^{\mu \nu} T_{\mu \nu}^{m}$. The two stress tensors $T_{\mu \nu}^{m}$ and $T_{\mu \nu}^{\varphi}$ are not separately conserved due to coupling of the scalar field $\varphi$ with matter. This can be seen by applying the Bianchi identities $\nabla^{\mu} G_{\mu \nu}=0$ to (2) which results in

$$
\nabla^{\mu} T_{\mu \nu}^{m}=-\nabla^{\mu} T_{\mu \nu}^{\varphi}=\frac{\beta(\varphi)}{M_{p}} \nabla_{\nu} \varphi T^{m}
$$

The parameter $\beta$ is generally a function of time. We will, however, restrict ourselves to the case that it can be regarded as a constant parameter. There are two important cases in which the function $\beta(\varphi)$ takes a constant configuration; $f(R)$ gravity and Brans-Dicke theory.

\section{$2.1 \quad f(R)$ Gravity}

The action for an $f(R)$ gravity theory in Jordan frame is given by [9]

$$
S_{J F}=\frac{1}{2} M_{p}^{2} \int d^{4} x \sqrt{-\bar{g}} f(\bar{R})+S_{m}\left(\bar{g}_{\mu \nu}, \psi\right)
$$

where $\bar{g}_{\mu \nu}$ is the metric in Jordan frame. A conformal transformation

$$
g_{\mu \nu}=A^{-2}(\varphi) \bar{g}_{\mu \nu}
$$

with $A^{-2}(\varphi) \equiv \frac{d f}{d R}=f^{\prime}(R)$ together with

$$
\varphi=\frac{M_{p}}{\beta} \ln A(\varphi)
$$

and $\beta=-\sqrt{\frac{1}{6}}$, transforms (9) into the action (1) with a potential [11] [12]

$$
V(\varphi(R))=\frac{M_{p}^{2}}{2}\left(\frac{R}{f^{\prime}(R)}-\frac{f(R)}{f^{\prime 2}(R)}\right)
$$

\subsection{Scalar-Tensor Gravity}

The general action of a scalar-tensor gravity is given by [13]

$$
S_{J F}=\frac{1}{16 \pi G} \int d^{4} x \sqrt{-\bar{g}}\left\{F(\phi) \bar{R}-Z(\phi) \bar{g}^{\mu \nu} \bar{\nabla}_{\mu} \phi \bar{\nabla}_{\nu} \phi-2 U(\phi)\right\}+S_{m}\left(\bar{g}_{\mu \nu}, \psi_{m}\right)
$$

where $F(\phi), Z(\phi)$ and $U(\phi)$ are some functions ${ }^{\dagger}$. This action is reduced to the action (1) by the conformal transformation (10) with $A(\varphi)=F^{-1 / 2}(\phi)$ and

$$
\left(\frac{d \varphi}{d \phi}\right)^{2}=2 M_{p}^{2}\left[\frac{3}{4}\left(\frac{d \ln F(\phi)}{d \phi}\right)^{2}+\frac{Z(\phi)}{2 F(\phi)}\right]
$$

\footnotetext{
†One can always redefine the scalar field to reduce $F(\phi)$ and $Z(\phi)$ to only one unknown function.
} 


$$
V(\varphi)=M_{p}^{2} U(\phi) F^{-2}(\phi)
$$

It is cleat from (14) that the coupling function $A(\varphi)$ depends on the functions $F(\phi), Z(\phi)$ and $U(\phi)$ through the relation (14). For some particular choices of these functions, $\beta$ takes a constant configuration and then, as a result of $(7), A(\varphi)$ takes an exponential form. This defines a class of scalar-tensor theories and we restrict ourselves to this class. One important theory in this class is given by $\mathrm{BD}$ parameterization in which $F(\phi)=16 \pi G \phi, Z(\phi)=16 \pi G \omega_{B D} / \phi$ and $U(\phi)=8 \pi G W(\phi)$, and then

$$
S_{J F}=\int d^{4} x \sqrt{-\bar{g}}\left(\phi \bar{R}-\frac{\omega_{B D}}{\phi} \bar{g}^{\mu \nu} \bar{\nabla}_{\mu} \phi \bar{\nabla}_{\nu} \phi-W(\phi)\right)+S_{m}\left(\bar{g}_{\mu \nu}, \psi\right)
$$

with $\omega_{B D}$ and $W(\phi)$ being BD parameter and the potential in Jordan frame, respectively. This action is reduced to (1) by [4] [14]

$$
\begin{gathered}
A(\varphi)=e^{\beta_{B D} \varphi / M_{p}} \\
\varphi(\phi) / M_{p}=\sqrt{\omega_{B D}+3 / 2} \ln \left(\frac{\phi}{\phi_{0}}\right) \\
V(\varphi)=W(\phi(\varphi)) e^{8 \beta_{B D} \varphi / M_{p}}
\end{gathered}
$$

where $\phi_{0} \sim G^{-1}$ and $\beta_{B D}=-1 / 2 \sqrt{\omega_{B D}+3 / 2}$. When $\omega_{B D} \rightarrow 0$, then $\beta_{B D} \rightarrow \frac{-1}{\sqrt{6}}$ and Einstein frame representations of $\mathrm{BD}$ model and $f(R)$ gravity are the same.

\section{Cosmological Setting}

We use a spatially flat homogeneous and isotropic cosmology described by Friedman-RobertsonWalker spacetime

$$
d s^{2}=-d t^{2}+a^{2}(t)\left(d x^{2}+d y^{2}+d z^{2}\right)
$$

where $a(t)$ is the scale factor. We also take $T_{\mu \nu}^{m}$ as the stress-tensor of a perfect fluid with energy density $\rho_{m}$ and pressure $p_{m}$. The Friedman equation is

$$
3 H^{2}=M_{p}^{-2}\left(\rho_{m}+\rho_{\varphi}\right)
$$

where $H=\frac{\dot{a}}{a}, \rho_{\varphi}=\frac{1}{2} \dot{\varphi}^{2}+V(\varphi)$ and overdot indicates differentiation with respect to time $t$. From (5) and (8), we obtain

$$
\begin{gathered}
\ddot{\varphi}+3 \frac{\dot{a}}{a} \dot{\varphi}+\frac{d V(\varphi)}{d \varphi}=-\frac{\beta(\varphi)}{M_{p}}\left(\rho_{m}-3 p_{m}\right) \\
\dot{\rho}_{m}+3 \frac{\dot{a}}{a}\left(\omega_{m}+1\right) \rho_{m}=Q \\
\dot{\rho}_{\varphi}+3 \frac{\dot{a}}{a}\left(\omega_{\varphi}+1\right) \rho_{\varphi}=-Q
\end{gathered}
$$


where

$$
Q=\frac{\beta(\varphi)}{M_{p}} \dot{\varphi}\left(\rho_{m}-3 p_{m}\right)
$$

is the interaction term, $\omega_{m} \equiv p_{m} / \rho_{m}$ and $\omega_{\varphi} \equiv p_{\varphi} / \rho_{\varphi}$. The direction of energy transfer depends on the sign of $Q$. For $Q>0$, the energy transfer is from the scalar field to the matter system and for $Q<0$, the reverse is true. The solution of equation (23) is

$$
\rho_{m}=\rho_{0 m} a^{-3\left(\omega_{m}+1\right)} e^{\frac{\left(1-3 \omega_{m}\right)}{M_{p}} \int \beta d \varphi}
$$

in which $\rho_{0 m}$ is an integration constant. This solution indicates that the evolution of energy density is modified due to interaction of $\varphi$ with matter. The expression (26) can be written as

$$
\rho_{m}=\rho_{0 m} a^{-3\left(\omega_{m}+1\right)+\epsilon}
$$

with $\epsilon$ being defined by

$$
\epsilon=\frac{\left(1-3 \omega_{m}\right) \int \beta d \varphi}{M_{p} \ln a}
$$

For constant $\beta,(28)$ reduces to

$$
\varphi=\sigma M_{p} \ln a
$$

with $\sigma$ being a constant defined by the relation $\epsilon=\beta \sigma\left(1-3 \omega_{m}\right)$. Here the parameter $\beta$ takes $\beta_{R}$ and $\beta_{B D}$ in $f(R)$ gravity and BD theory, respectively.

The expression (27) states that when $\epsilon>0$, matter is created and energy is constantly injecting into the matter so that the latter will dilute more slowly compared to its standard evolution $\rho_{m} \propto a^{-3\left(\omega_{m}+1\right)}$. Similarly, when $\epsilon<0$ the reverse is true, namely that matter is annihilated and the direction of energy transfer is outside of the matter system so that the rate of dilution is faster than the standard one.

Let us first take the matter system to be vacuum energy density characterized by a perfect fluid with equation of state parameter $\omega_{m}=-1$. Thus the vacuum energy density is anomalously coupled to gravity. In this case, $(27)$ becomes $\rho_{\Lambda} \equiv \rho_{m}=\rho_{0 \Lambda} a^{\epsilon}$ where we have set $\rho_{0 m}=\rho_{0 \Lambda}$. In an expanding universe, the requirement that a large vacuum energy density $\rho_{\Lambda 0}$ reduces during the expansion needs $\varepsilon<0$. Note that in this case the direction of energy transfer is out of vacuum. We can use Friedman equation (21) and the equations (23) and (24) to write the deceleration parameter

$$
q(z)=-1-\frac{\dot{H}}{H^{2}}=-1-\frac{\frac{1}{2}\left[\epsilon \Omega_{\Lambda}-\frac{Q}{H \rho_{c}}-3\left(\omega_{\varphi}+1\right) \omega_{\varphi}\right]}{\left(\Omega_{\Lambda}+\Omega_{\varphi}\right)}
$$

where $\Omega_{\Lambda}=\rho_{\Lambda} / \rho_{c}, \Omega_{\varphi}=\rho_{\varphi} / \rho_{c}$ and $\rho_{c}=3 H_{0}^{2} M_{p}^{2}$ is the critical density. On can use (25) to show that $\frac{Q}{H \rho_{c}}=\epsilon \Omega_{\Lambda}$. This together with spatial flatness, which requires that $\Omega_{\Lambda}+\Omega_{\varphi}=1$, reduce $(30)$ to

$$
q(z)=-1+\frac{3}{2}\left(\omega_{\varphi}+1\right)\left(1-\Omega_{\Lambda}\right)
$$

which accelerating expansion requires that $\omega_{\varphi}+1<\frac{2}{3\left(1-\Omega_{\Lambda}\right)}$.

The coincidence problem concerns with the constancy of the ratio $r=\frac{\rho_{m}}{\rho_{c}}$. Using (23) and (24), we can write

$$
\dot{r}=r H\left[\epsilon(r+1)+\left(\omega_{\varphi}-\omega_{m}\right)\right]
$$


where we have used $Q=\epsilon H \rho_{m}$. If we set $\omega_{m}=0$, the constancy of $r$ requires that $\omega_{\varphi}=$ $-\frac{1}{3} \epsilon(r+1)$ which $\omega_{\varphi}>1$ for a vacuum decay $(\epsilon<0)$.

\section{Conclusion}

There is no observational constraints on gravitational coupling of matter systems during expansion of the universe such as baryons, radiation, vacuum, dark matter or dark energy. The weak equivalence principle which is supported by recent observations [16] only constrains the baryons. Thus it is quite possible that other kinds of matter systems such as vacuum couples differently with gravity.

We consider a dynamical mechanism which works with expansion of the universe and has its origin in gravitational coupling of the vacuum. We assume that vacuum couples anomalously with gravity in the sense that the metrics of the gravitational and matter parts are not the same but conformally related. The conformal factor is then controlled by a dynamical scalar field. We provide some examples from $f(R)$ gravity and BD theories. We have shown that the cosmological constant problem may be alleviated in such theories due to anomalous gravitational coupling of vacuum.

\section{References}

[1] S. Weinberg, Rev. Mod. Phys. 61, 1 (1989)

[2] S. M. Carroll, Living Rev. Rel. 4, 1 (2001)

T. Padmanabhan, Phys. Rept. 380, 235 (2003)

[3] Y. Bisabr, Phys. Rev. D 82 124041, (2010)

[4] H. Salehi and Y. Bisabr, Class. Quant. Grav. 19, 2369 (2002)

Y. Bisabr, Gen. Rel. Grav. 42, 1211 (2010)

[5] J. Khoury and A. Weltman, Phys. Rev. D 69, 044026 (2004)

J. Khoury and A. Weltman, Phys. Rev. Lett. 93, 171104 (2004)

[6] Y. Bisabr, Phys. Lett. B 683, 96 (2010)

[7] Ph. Brax, C. van de Bruck, A. Davis, J. Khoury and A. Weltman, Phys. Rev. D 70, $123518(2004)$

A. Upadhye, S. S. Gubser and J. Khoury, Phys. Rev. D 74, 04024 (2006)

Ph. Brax, C. van de Bruck, A. Davis and D. J. Shaw , Phys. Rev. D 78, 104021 (2008)

Ph. Brax and K. Zioutas, Phys. Rev. D 82, 043007 (2010)

[8] S. M. Carroll, V. Duvvuri, M. Trodden and M. S. Turner, Phys. Rev. D 70, 043528, (2004) 
[9] S. M. Carroll, A. De Felice, V. Duvvuri, D. A. Easson, M. Trodden and M. S. Turner, Phys. Rev. D 71, 063513, (2005)

G. Allemandi, A. Browiec and M. Francaviglia, Phys. Rev. D 70, 103503 (2004)

X. Meng and P. Wang, Class. Quant. Grav. 21, 951 (2004)

M. E. soussa and R. P. Woodard, Gen. Rel. Grav. 36, 855 (2004)

S. Nojiri and S. D. Odintsov, Phys. Rev. D 68, 123512, (2003)

[10] C. Brans and R. H. Dicke, Phys. Rev. 124, 925 (1961)

[11] G. Magnano and L. M. Sokolowski, Phys. Rev. D 50, 5039 (1994)

[12] K. Maeda, Phys. Rev. D 39, 3159 (1989)

D. Wands, Class. Quant. Grav. 11, 269 (1994)

[13] G. Esposito-Farese and D. Polarski, Phys. Rev. D 63, 063504 (2001)

[14] V. Faraoni, Cosmology in Scalar-Tensor Gravity. Kluwer Academic, Norwell (2004)

[15] A. G. Riess, et al., Astrophys. J. 560, 49 (2001)

T. Padmanabhan and T. Roy Choudhury, Mon. Not. Roy. Astron. Soc.344, 823 (2003)

L. Amendola, Mon. Not. R. Astron. Soc. 342, 221 (2003)

[16] C. M. Will, Theory and Experiment in Gravitational Physics, (Cambridge University Press, 3rd edition, Cambridge, 1993)

C. M. Will, Liv. Rev. Rel. 9, 3 (2005) 